\title{
The Study Isobaric 0.75\% Ropivacaine 3.5ml (26.25 mg) Intrathecally for Lower Limb Surgeries to Evaluate Perioperative Hemodynamic Stability
}

\author{
Kunwar Singh Thakur ${ }^{1}$, Utsav Sharma ${ }^{1}$, Preeti Goyal ${ }^{2}$, Bhanu Choudhary ${ }^{3}$ \\ ${ }^{1}$ Senior Resident, Department of Anaesthesiology, Gajra Raja Medical College, Gwalior, Madhya Pradesh, India, ${ }^{2}$ Professor, Department of \\ Anaesthesiology, Gajra Raja Medical College, Gwalior, Madhya Pradesh, India, ${ }^{3}$ Professor and Head, Department of Anaesthesiology, Gajra Raja Medical \\ College, Gwalior, Madhya Pradesh, India.
}

\section{Abstract}

Background: The purpose of the study is Isobaric $0.75 \%$ Ropivacaine $3.5 \mathrm{ml}$ (26.25mg) Intrathecally for Lower Limb Surgeries to Evaluate Perioperative Hemodynamic. Fifty patients admitted to our hospital undergoing lower extremity surgeries under spinal anesthesia. Subjects and Methods: Pre-anesthetic assessment was done prior to the day of surgery which included past history of chronic illness and medication, drug therapy (especially corticosteroids, anti hypertensive, anti coagulant, anti diabetic, anti convulsant medications), drug sensitivity and past anesthetic experience along with routine investigations including, haemoglobin, random blood sugar, serum urea, serum creatinine, and serum electrolytes, chest X-ray, ECG. Fifty patients admitted to our hospital undergoing lower extremity surgeries under spinal anesthesia included in the study. Results: Patients in the age group 18 - 60 years were selected including 45 males and 5 females. Thirty-Nine patients had an ASA grade I and 11 patients had an ASA grade II. The duration of surgeries ranged from 45 to 270 minutes \& there were no significant changes in the pulse rate perioperatively. Conclusion: A study was conducted in 50 patients belonging to ASA grade I and II posted for lower limb surgeries. They received $3.5 \mathrm{ml}$ of $0.75 \%$ isobaric Ropivacaine in L2-3 interspace. All patients were preloaded with $1000 \mathrm{ml}$ of ringer lactate. Following institution of subarachoid block sensory characteristics such as onset of sensory block, maximum level achieved, duration of sensory block and request for first dose of rescue analgesics were studied. We found the requirement for postoperative analgesics was 218.97 minutes. Hemodynamic parameters were stable in most of the patients. Postoperative side effects included shivering, nausea. Thus we conclude that isobaric $0.75 \%$ ropivacaine $26.25 \mathrm{mg}, 3.5 \mathrm{ml}$ when administered intrathecally in L2-3 interspace provides an adequate sensory block for lower limb surgeries, which makes it a good choice for lower limb surgeries with benefits of early ambulation.

Keywords: Ropivacaine, Intrathecally, Lower Limb \& Surgeries.

Corresponding Author: Dr. Utsav Sharma, Senior Resident, Department of Anaesthesiology, Gajra Raja Medical College, Gwalior, Madhya Pradesh, India.

Email: varuny.indore09@gmail.com

Received: June 2020

Accepted: June 2020

\section{Introduction}

Bupivacaine aminoamide local anesthetic was first described by A Ekenstam in 1957. It has been used successfully for spinal anesthesia in either a glucose-free or a hyperbaric solution. It produces sensory and motor block for a longer duration. It is still widely used but has been associated with fatal cardiac and neurological toxicity when given intravascularly accidentally. ${ }^{[1]}$

Ropivacaine is a long-acting regional anesthetic drug that is structurally related to Bupivacaine. Bupivacaine, which is a racemate, developed for the purpose of reducing potential toxicity and improving relative sensory and motor block profiles. It provides long-lasting block. The reduced toxic potential is strongly supported by animal and volunteer studies, resulting not only in higher plasma concentrations and doses before signs of systemic toxicity but also in no cardiovascular toxicity or minimal signs of cardiac effects after CNS toxicity occurs. ${ }^{[2]}$

Ropivacaine shows a more reliable differentiation between sensory and motor block, making early mobilization possible. Extensive clinical data have shown that it has been used safely and effectively for local infiltration, epidural and peripheral nerve blocks. However, the experience of intrathecal anesthesia with ropivacaine is not documented. In vivo studies in dogs and mice have indicated that, at equal drug concentrations, ropivacaine was less potent and had a shorter duration of a motor block than bupivacaine after spinal administration. ${ }^{[3]}$

One of the most essential properties of long-acting local anesthesia is to reversibly inhibit the nerve impulses, thus causing a prolonged sensory or motor blockade appropriate in different types of surgeries. The acute pain relief obtained at lower doses in postoperative and labor patients due to sensory blockade is sometimes marred by accompanying 
motor blockade, which serves no purpose and is quite undesirable. ${ }^{[4]}$

The clinical efficacy and safety of $3.5 \mathrm{~mL} 0.75 \%$ (26.25 mg.) glucose-free ropivacaine as a local anesthetic for spinal anesthesia in patients undergoing lower limb surgeries.

\section{Subjects and Methods}

This study was conducted at Gajra Raja Medical College, Gwalior from Jul 2018 to June 2019, after approval from the ethical committee. Fifty patients admitted to our hospital undergoing lower extremity surgeries under spinal anesthesia included in the study.

Pre-anesthetic assessment was done prior to the day of surgery which included past history of chronic illness and medication, drug therapy (especially corticosteroids, anti hypertensive, anti coagulant, anti diabetic, anti convulsant medications), drug sensitivity and past anesthetic experience along with routine investigations including, haemoglobin, random blood sugar, serum urea, serum creatinine, and serum electrolytes, chest X-ray, ECG.

\section{$\underline{\text { Inclusion Criteria }}$}

1. Age- 18-60 years

2. ASA Grade - I and II

3. Lower limb surgeries

4. Valid informed consent

\section{Exclusion Criteria}

1. Hematologic disease.

2. Bleeding and coagulation abnormalities

3. Cardiac and respiratory diseases

4. History of allergy to local anesthetics

5. Pregnancy, Pediatric age group, Geriatric age group

6. Local infection in the lumbar region

The pre-anesthetic assessment was done before the day of surgery which included in the history of chronic illness and medication, drug therapy (especially corticosteroids, antihypertensive, anticoagulant, anti-diabetic, anticonvulsant medications), drug sensitivity and past anesthetic experience along with routine investigations including, hemoglobin, random blood sugar, serum urea, serum creatinine, and serum electrolytes, chest X-ray, ECG.

Informed and written consent after explaining the process to the patients. A thorough general, physical examination of all systems was carried out. The nature of the procedure was explained to the patients, informed and written consent.

\section{Results}

Table 1: Demographic data
\begin{tabular}{|l|l|}
\hline Parameters & No. of patientsn = 50 (100\%) \\
\hline Age (mean) & $18-60 \quad(34.52)$ \\
\hline Sex (M:F) & $45: 5 \quad(90: 10)$ \\
\hline ASA Grade (I:II) & $39: 11 \quad(78: 22)$ \\
\hline Duration of surgery (in mins) & $45-270(114.66$ mins)(S.D. 49.33) \\
\hline
\end{tabular}

Patients in the age group $18-60$ years were selected including 45 males and 5 females. Thirty-Nine patients had an ASA grade I and 11 patients had an ASA grade II. The duration of surgeries ranged from 45 to 270 minutes.

\section{Perioperative hemodynamic monitoring}

Table 2: Perioperative pulse rate changes

\begin{tabular}{|l|l|l|}
\hline Time (in mins) & Pulse rate (Mean) & S.D \\
\hline 0 & 92.86 & 17.50 \\
\hline 2 & 95.08 & 17.84 \\
\hline 5 & 92.14 & 15.57 \\
\hline 10 & 90.54 & 14.58 \\
\hline 15 & 89.86 & 15.88 \\
\hline 20 & 86.88 & 15.11 \\
\hline 25 & 86.28 & 15.98 \\
\hline 30 & 85.46 & 14.26 \\
\hline 45 & 85.36 & 14.60 \\
\hline 60 & 86.14 & 15.31 \\
\hline 120 & 84.82 & 12.94 \\
\hline 180 & 86.79 & 12.50 \\
\hline 240 & 85.23 & 12.23 \\
\hline 300 & 86.84 & 13.44 \\
\hline 360 & 80.37 & 8.9 \\
\hline
\end{tabular}

There were no significant changes in the pulse rate perioperatively.

Eight patients had intraoperative hypotension was managed by mephentermine $6 \mathrm{mg}$. Bradycardia occurred in 3 patients, they were given atropine sulfate $0.6 \mathrm{mg}$ IV. None of the patients had hypotension or bradycardia in the post-operative period. Two patients had intraoperative shivering. Postoperatively complications like shivering, nausea and headache were reported in one patient each.

Table 3: Perioperative systolic pressure changes

\begin{tabular}{|l|l|l|}
\hline Time (in mins) & Systolic pressure (Mean) & S.D. \\
\hline 0 & 123.78 & 13.12 \\
\hline 2 & 124.4 & 14.23 \\
\hline 5 & 118.26 & 12.95 \\
\hline 10 & 115.82 & 12.63 \\
\hline 15 & 115.44 & 13.02 \\
\hline 20 & 117.04 & 14.51 \\
\hline 25 & 114.76 & 12.76 \\
\hline 30 & 115.46 & 14.65 \\
\hline 45 & 115.86 & 14.25 \\
\hline 60 & 116.74 & 12.48 \\
\hline 120 & 116.82 & 11.78 \\
\hline 180 & 117.79 & 10.64 \\
\hline 240 & 119 & 7.21 \\
\hline 300 & 119.69 & 8.1 \\
\hline 360 & 117.75 & 7.36 \\
\hline
\end{tabular}

Table 4: Perioperative diastolic pressure changes

\begin{tabular}{|l|l|l|}
\hline Time (in mins) & Diastolic pressure (Mean) & S.D. \\
\hline 0 & 76.72 & 10.34 \\
\hline 2 & 76.16 & 9.19 \\
\hline 5 & 73.3 & 10.19 \\
\hline 10 & 71.96 & 12.68 \\
\hline 15 & 73.12 & 10.80 \\
\hline 20 & 72.42 & 10.19 \\
\hline 25 & 71.72 & 10.60 \\
\hline 30 & 70.6 & 11.06 \\
\hline 45 & 72.76 & 10.21 \\
\hline 60 & 73.26 & 8.9 \\
\hline 120 & 75.12 & 7.5 \\
\hline 180 & 75.54 & 7.13 \\
\hline 240 & 75.31 & 6.60 \\
\hline 300 & 77.07 & 5.07 \\
\hline 360 & 76.62 & 4.43 \\
\hline
\end{tabular}




\section{Discussion}

Mc Namee et al. ${ }^{[5]}$ found both intrathecalropivacaine and bupivacaine produced an initial moderate fall in the arterial pressure keeping with the expected sympathetic block produced by spinal anesthesia. Intraoperative hypotension requiring ephedrine occurred in $12 \%$ and $26 \%$ respectively of patients. Two patients in the ropivacaine group also required atropine. Hemodynamic changes were similar, no significant difference in the hemodynamic parameters was observed in the first 15 minutes. ${ }^{[5]}$ Various other authors like Helena et al., Ph E. Gautier et al., Y.Y Lee et al. in their respective comparisons between the isobaric preparations of the drugs found no significant difference in the incidences of hypotension and bradycardia. ${ }^{[6-8]}$ It was found that these effects were related to the level of sympathetic block produced rather than the drug characteristic. J.B. Whiteside et al. ${ }^{[9]}$ found significant differences $(p=0.001)$. In their comparative study of $0.5 \%$ hyperbaric ropivacaine with hyperbaric $0.5 \%$ bupivacaine with hypotension occurring in the bupivacaine group as compared to $15 \%$ in the ropivacaine group. ${ }^{[9]}$

Table 5: Number of Patients with Intraoperative and postoperative complications

\begin{tabular}{|l|l|l|}
\hline Complications & $\begin{array}{l}\text { Intraoperative } \\
(\mathbf{n = 5 0 )}\end{array}$ & $\begin{array}{l}\text { Postoperative } \\
(\mathbf{n = 5 0 )}\end{array}$ \\
\hline Hypotension & 8 & 0 \\
\hline Bradycardia & 3 & 0 \\
\hline Nausea and Vomiting & 0 & 1 \\
\hline Shivering & 2 & 1 \\
\hline Headache & 0 & 1 \\
\hline
\end{tabular}

Lopez-Soriano et al. in comparative study found fewer episodes of hypotension ( $0 \%$ vs $17.7 \%)$ and bradycardia $(4.4 \%$ vs $8.8 \%)$ in hyperbaric $0.5 \%$ ropivacaine in comparison to $0.5 \%$ hyperbaric bupivacaine. ${ }^{[10]}$

In our study 8 patients were hypotension was seen intraoperatively which was treated a single dose of phentermine sulfate $6 \mathrm{mg}$, Bradycardia in 03 patients, they were given $0.6 \mathrm{mg}$. atropine sulfate IV.

\section{Conclusion}

A study was conducted in 50 patients belonging to ASA grade I and II posted for lower limb surgeries. They received $3.5 \mathrm{ml}$ of $0.75 \%$ isobaric Ropivacaine in L2-3 interspace. All patients were preloaded with $1000 \mathrm{ml}$ of ringer lactate. Following institution of subarachoid block sensory characteristics such as onset of sensory block, maximum level achieved, duration of sensory block and request for first dose of rescue analgesics were studied.

We found a requirement for postoperative analgesics was 218.97 minutes. Hemodynamic parameters were stable in most of the patients. Postoperative side effects included shivering, nausea. Thus we conclude that isobaric $0.75 \%$ ropivacaine $26.25 \mathrm{mg}, \quad 3.5 \mathrm{ml}$ when administered intrathecally in L2-3 interspace provides an adequate sensory block for lower limb surgeries, which makes it a good choice for lower limb surgeries with benefits of early ambulation.

\section{References}

1. Aberg G. Toxicological and local anesthetic effects of optically active isomers of two local anesthetic compounds. ActaPharmacolToxicolScand 1972;31:273-86.

2. Boztug N, Bigat Z, Karsli B, Saykal N. Comparison of ropivacaine and bupivacaine for intrathecal anesthesia during outpatient arthroscopic surgery. J. ClinAnesth 2006;18(7):521-5.

3. Carvalho AC, Machado JA, Nociti JR: Spinal anesthesia with $0.5 \%$ hyperbaric ropivacaine and $0.5 \%$ hyperbaric bupivacaine: a comparative study. Rev Bras Anesthesiol. 2002; 52(6):659-65.

4. M. Mantouvalou, S. Ralli, H. Arnaoutoglou, G. Tziris, G. Papadopoulos. Spinal anesthesia: Comparison of plain ropivacaine, bupivacaine and levobupivacaine for lower abdominal surgery. ActaAnaesthBelg 2008;59:65-71

5. D.A. Mc Namee, A.M. McClelland, S. Scott, K.R. Milligan, L. Westman, U. Gustafsson. Spinal anesthesia: comparison of plain ropivacaine $5 \mathrm{mg} / \mathrm{ml}$ with bupivacaine $5 \mathrm{mg} / \mathrm{ml}$ for major orthopedic surgery. British Journal of Anesthesia 2002;89(5):702-6.

6. Helena Kallio, Eljais-Veli T. Snail, Markku P. Kero, Per H. Rosenberg. A comparison of intrathecal plain solutions containing ropivacaine 20 or 15 $\mathrm{mg}$ versus bupivacaine $10 \mathrm{mg}$. AnesthAnalg 2004;99:713-7.

7. Ph. E. Gautier, M. De Kock, Van Steenberg, N. Poth, B. Lahaye-Goffart, L. Fanard, J. L. Hody. Intrathecalropivacaine for ambulatory surgery: a comparison between intrathecal bupivacaine and intrathecalropivacaine for knee arthroscopy. Anesthesiology 1999; 91:1239-45.

8. Y.Y. Lee, W.D. NganKee, K. Muchhal, C.K. Chan: Randomized doubleblind comparison of bupivacaine-fentanyl and bupivacaine-fentanyl for spinal anesthesia for urological surgery. ActaAnaesthesiolScand 2005; 49:1477-1482.

9. J. B. Whiteside, D. Burke, J. A. W. Wildsmith, Comparison of ropivacaine $0.5 \%$ (in glucose $5 \%$ ) with bupivacaine $0.5 \%$ (in glucose $8 \%$ ) for spinal anesthesia for elective surgery. British Journal of Anesthesia 2003; 90(3):304-8.

10. Lopez- Soriano F, Lajarin B, Rivas F, Verdu JM, Lopez-Robles J: Hyperbaric subarachnoid ropivacaine in ambulatory surgery: a comparative study with hyperbaric bupivacaine. Rev EspAnestesiolReanim. 2002;49(2):71-5.

Copyright: (c) the author(s), 2020. It is an open-access article distributed under the terms of the Creative Commons Attribution License (CC BY 4.0), which permits authors to retain ownership of the copyright for their content, and allow anyone to download, reuse, reprint, modify, distribute and/or copy the content as long as the original authors and source are cited.

How to cite this article: Thakur KS, Sharma U, Goyal P, Choudhary B. The Study Isobaric 0.75\% Ropivacaine 3.5ml (26.25mg) Intrathecally for Lower Limb Surgeries to Evaluate Perioperative Hemodynamic Stability. Acad. Anesthesiol. Int. 2020;5(1):168-170.

DOI: dx.doi.org/10.21276/aan.2020.5.1.35

Source of Support: Nil, Conflict of Interest: None declared. 